\title{
Scoring system for papillary thyroid carcinoma - a histopathological study
}

\author{
Prabhu S.C. ${ }^{1}$, Umashankar T. ${ }^{2}$ \\ ${ }^{1}$ Dr. Swathi C. Prabhu, Assistant Professor, Department of Pathology, Melaka Manipal Medical College, Manipal, \\ Karnataka, ${ }^{2}$ Dr. Umashankar T., Professor, Department of Pathology, Father Muller Medical College, Mangalore, \\ Karnataka, India.
}

Corresponding Author: Dr. Swathi C. Prabhu, Manjunath Krupa, Temple Road, Saralebettu, Manipal, Udupi District Karnataka, India. E-mail: prabhuswathi15@gmail.com

\begin{abstract}
Background: Cancer of the thyroid gland is the most common endocrine malignant neoplasm, and papillary thyroid carcinoma (PTC) accounts for majority of the cases. PTC cases are identified by its distinct histopathological features. Due to lack of definable criteria and overlapping features seen in other diseases of thyroid giving rise to false positive cases and errors in diagnosis may reflect drastically on the prognosis and treatment outcome. Objective: To analyse the frequency of PTC microscopic criteria in a series of benign lesions of thyroid and PTC cases and to build an optimal cut off score which could help us differentiate between these cases. Materials and Methods: The study included 86 thyroid lesions, 43 benign and 43 PTC cases. Histopathological parameters analysed included colloid, papillae, nuclear crowding, nuclear grooving, nuclear enlargement, oval nucleus, nuclear clearing. Results: The above mentioned parameters could differentiate between benign and PTC cases. Based on sensitivity and specificity, the cut off derived for every parameter is as follows: colloid $<50 \%$, papillae $>1 \%$, nuclear crowding $>25 \%$, nuclear grooving $>5 \%$, nuclear enlargement $>50 \%$, ovoid nucleus $>25 \%$, nuclear clearing $>50 \%$. The scoring system was built based on these parameters. The score proposed helped us to classify benign and PTC cases. PTC cases scored a total score of $>7$ indicating that seven can be considered as cut off value to differentiate between benign and PTC cases. Conclusion: Definite distinction between benign and PTC cases was possible when all the parameters were clubbed together with an important role in cases with diagnostic dilemma.
\end{abstract}

Key words: Microscopic criteria, Adenomatous nodule, Follicular adenoma, papillary thyroid carcinoma, Thyroid.

\section{Introduction}

papillary thyroid carcinoma (PTC) is the most common malignant neoplasm of thyroid accounts for $80-85 \%$ of the cases. The most common etiologic factor is radiation, but genetic susceptibility and other factors also contribute to the development of PTC. Generally, PTC has a favourable prognosis, with long-term survival rates greater than $90 \%$ particularly in patients younger than 45 years $[1,2]$.

Right diagnosis and appropriate treatment helps in achieving desired results. PTCs are identified by its distinct histopathological features such as papillary architecture, nuclear clearing, ground glass or Orphan Annie-eyed appearance, oval shaped nuclear enlargement, powdery chromatin, intranuclear cytoplasmic inclusions, nuclear grooving, and nuclear

Manuscript received: $8^{\text {th }}$ December 2018

Reviewed: $18^{\text {th }}$ December 2018

Author Corrected: $26^{\text {th }}$ December 2018

Accepted for Publication: $31^{\text {st }}$ December 2018 overlapping [3]. In India, as per Mumbai Cancer Registry, age adjusted incidence rates are about 1 for males and 1.8 for females per 100,000 populations. Frequency of thyroid cancer among all cancer cases was $0.1 \%-0.2 \%$ [4]. There has been substantial increase in incidence rates due to awareness and increase in early detection by MRI and CT of non-palpable nodules. Papillary micro-carcinomas (less than $1 \mathrm{~cm}$ size) have an excellent prognosis $[3,5]$.

Most commonly PTC presents as a solitary nodule or as cervical lymphadenopathy, cold nodule on radioactive iodine scan. Fine needle aspiration of thyroid lesion or lymph node helps in the initial diagnosis preoperatively. Grossly they present as grey white masses with infiltrative borders. Some may present with cystic change and some with calcified areas. The lymphatics, being the most common route of metastasis and hence these tumours might present as cervical 


\section{Original Research Article}

lymphadenopathy $[1,6]$. Histopathology remains as a gold standard for diagnosis of PTC. It is characterised by its distinct nuclear features which include, nuclear enlargement, nuclear overlapping, oval nucleus, nuclear grooves, and nuclear inclusions. Papillae, psammoma bodies, thick colloid, multinucleated cells are also noted [7].

Papillae are also noted in nodular goitre, follicular adenoma, diffuse hyperplasia and are usually not seen in follicular variant of PTC. Nuclear features though focally are noted in a variety of lesions including adenomatous nodules, Hashimoto's thyroiditis.

These cases may also show areas of nuclear clearing, nuclear crowding, grooving. These overlapping features pose difficulties in diagnosis and are the reasons for false positive cases which significantly influence treatment and prognosis [3].

The objectives of this study

- To analyse, the percentage of tumour cells with each microscopic criterion of PTC proposed in individual tumour, and the frequency of PTC microscopic criteria in a series of 86 cases. To determine the optimal cut off for every parameter based on sensitivity and specificity.

- To propose an optimal cut off score and to categorise the thyroid tumours in to benign and PTC based on this score.

\section{Materials and Methods}

Place of study: The present study was conducted in Father Muller medical college, tertiary care centre from May 2013 to October 2016.

Type of study: Comparative study between benign and PTC cases.

Sampling method: Retrospective study 43 benign and 43 PTC cases were studied.

Inclusion criteria: Malignant cases included classical and follicular variant of PTC (FVPTC) $(>1 \mathrm{~cm})$.Benign cases included were follicular adenoma, Hurthle cell adenoma and adenomatous nodule.

Exclusion criteria: Rest of the variants of PTCs, microcarcinomas $(<1 \mathrm{~cm})$ and other malignant lesions of thyroid were excluded from our study. Inflammatory, autoimmune, granulomatous conditions were also excluded. These cases were retrieved from archives.

Statistical method: The parameters were compared between benign and PTC cases. Comparisons of frequencies among the two groups were performed using the Chi-square test or Fisher exact test when necessary.

Secondly, sensitivity, specificity was determined at each cut off level that was designed. The cut offs and the corresponding scores derived was then applied to benign thyroid lesions and PTC cases

Method and scoring system: Morphometric analysis was performed on paraffin embedded Hematoxylin and Eosin ( $\mathrm{H}$ $\&$ E) sections and the following parameters were formulated and evaluated and scored accordingly as shown below.

A semi quantitative microscopic analysis in four categories $(\leq 25 \% ;>25 \%$ and $\leq 50 \% ;>50 \%$ of tumour) was assessed for the following parameters: Colloid, nuclear crowding, nuclear enlargement, oval nucleus.

Papillae were scored as $<1 \%$ and $>1 \%$ and nuclear grooving as $<5 \%$ and $>5 \%$. 10 high power fields were evaluated in 10 consecutive fields with a gap maintained between the two fields to avoid overlapping.

Six to 10 nuclei when seen closely packed together, was considered as nuclear crowding.

Nuclear enlargement was considered when the size was four to six times of the size of small lymphocyte. Nuclear clearing was estimated by counting number of cells showing clearing per high power field and multiplying by area in percentage (Table 1).

The involvement was considered focally when the area involved was $<25 \%$. 


\section{Original Research Article}

Table-1: Grading of various parameters.

\begin{tabular}{|c|l|c|}
\hline Parameters & Grade & Score \\
\hline Colloid & $<25 \%$ & 2 \\
& $25-50 \%$ & 1 \\
\hline Papillae & $>50$ & 1 \\
& $<1 \%$ & 2 \\
\hline Nuclear crowding & $>1 \%$ & 1 \\
\hline Area in \% & $<25 \%$ & 2 \\
\hline Nuclear grooving & $25-50 \%$ & 3 \\
\hline Area in \% & $>50 \%$ & 2 \\
\hline Nuclear enlargement & $<5 \%$ & 1 \\
\hline Area in \% & $>5 \%$ & 2 \\
& $<25 \%$ & 3 \\
\hline Oval nucleus & $25-50 \%$ & 1 \\
Area in \% & $>50 \%$ & 2 \\
& $<25 \%-1$ & 3 \\
\hline Nuclear clearing & $25-50 \%-2$ & $1,2,3,4,6,9$ \\
\hline
\end{tabular}

After measurement, the data were transferred to MS-Excel sheet for further analysis. These parameters were compared between benign and PTC cases. Comparisons of frequencies among the two groups were performed using the Chi-square test or Fisher exact test when necessary. Secondly, sensitivity, specificity was determined at each cut off level that was designed. The cut offs and the corresponding scores derived was then applied to benign thyroid lesions and PTC cases. The score proposed is given below (Table 2).

Table-2: Cut offs and corresponding scores on each microscopic criterion

\begin{tabular}{|l|c|c|}
\hline \multicolumn{1}{|c|}{ Parameter } & Absent & Present \\
\hline Colloid $(<50 \%)$ & 0 & 2 \\
\hline Papillae $(>1 \%)$ & 0 & 2 \\
\hline Nuclear crowding $(>25 \%)$ & 0 & 2 \\
\hline Nuclear grooving $(>5 \%)$ & 0 & 3 \\
\hline Nuclear enlargement $(>50 \%)$ & 0 & 2 \\
\hline Oval nucleus $(>25 \%)$ & 0 & 4 \\
\hline Nuclear clearing $(>50 \%)$ & 0 & 2 \\
\hline
\end{tabular}

For all statistical tests, a $p$ value of less than 0.05 was considered to indicate a significant difference.

\section{Results}

Out of 86 cases, $18(20.8 \%)$ were FVPTC and 25(28.7\%) were classic variants. Benign cases studied were 32(37.9\%) cases of adenomatous nodule, $5(5.7 \%)$ cases of follicular adenoma and 6(6.9\%) cases of Hurthle cell adenoma.

The presence, the frequency of each PTC microscopic criterion, was evaluated in PTC, according to the percentage of tumour with the microscopic criteria (Table 3). A quantitative assessment was done on benign and PTC cases. 
Original Research Article

Table-3: Frequency of PTC microscopic criteria in PTC and benign thyroid lesions.

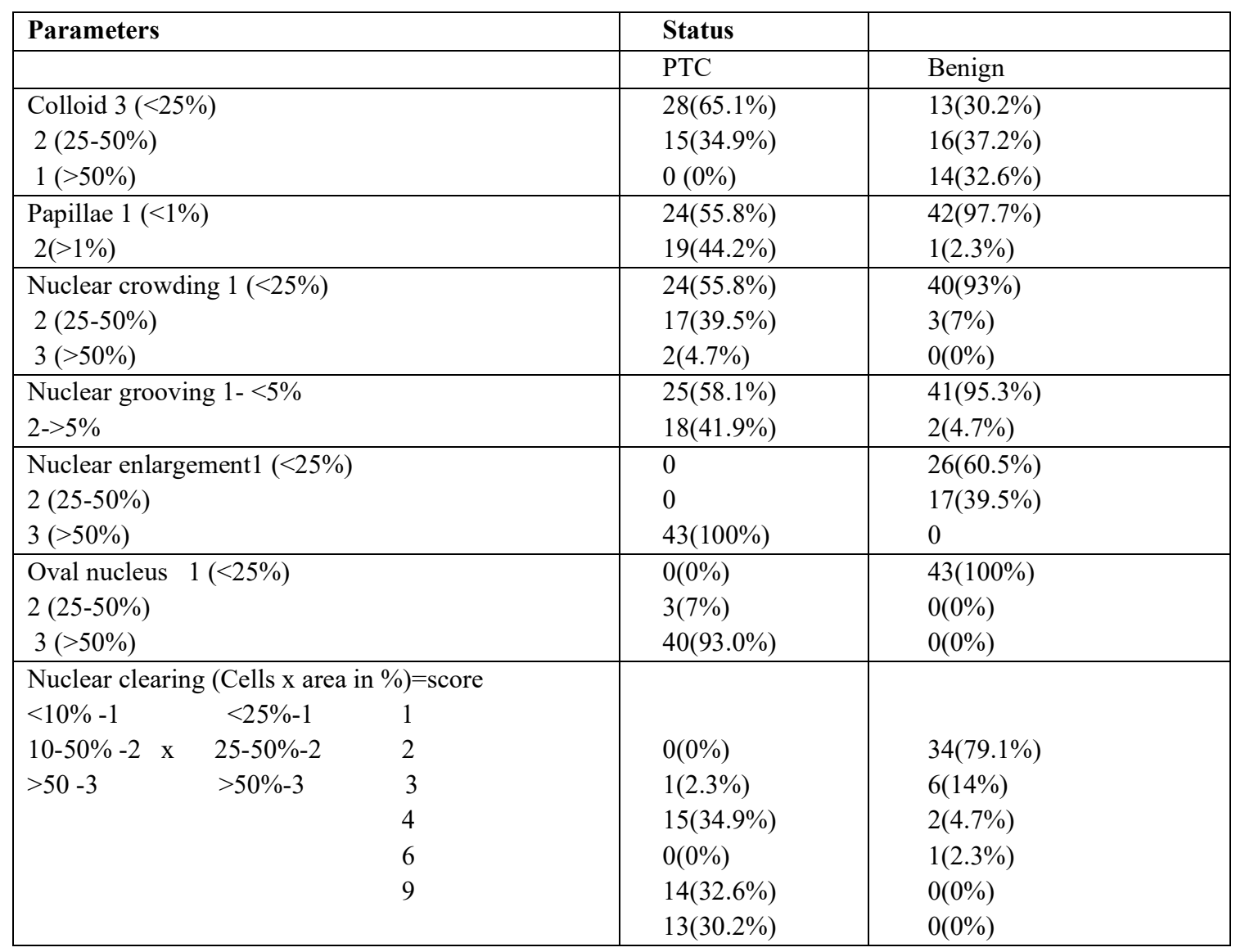

Seventy percentage of the benign cases showed colloid status $>25 \%$ of the area in contrast to malignant cases which constituted $34.9 \%$ cases. Papillae, one of the most important parameter in classical variant of PTC (55.8\%) were seen only in one (2.3\%) of the case in the benign category. Enlarged nuclei constituting $>50 \%$ area was seen in almost all PTC cases in contrast to benign cases were nuclear enlargement was observed focally ( $<25 \%$ area) in $60.5 \%$ cases. Majority of the benign cases(93\%) showed focal area of nuclear crowding compared to malignant cases which were distributed between focal(55.8\%) and diffuse (44.2\%). About $93 \%$ of the malignant cases showed diffuse oval enlargement of the nucleus ( $>50 \%$ of the area) compared to benign cases which showed focal enlargement.

Secondly, based on the $p$ value, the threshold value was determined for the criteria to allow us a clear distinction between benign and PTC cases. The threshold value derived was $50 \%$ for colloid status, nuclear crowding, nuclear enlargement, nuclear clearing, $25 \%$ for oval nucleus, $5 \%$ for nuclear grooving, and $1 \%$ for papillae. These criteria defined were significantly more frequently observed in PTC than benign cases $(p<0.05)$. Sensitivity and specificity, $p$ value, was determined at the level of cut off and the results are as follows. $P$ value was considered significant if $<0.05$ and highly significant when $<0.001$.

Table-4: Sensitivity, specificity and $p$ value at the level of optimal cut off.

\begin{tabular}{|l|c|c|c|c|c|}
\hline Parameter & Sensitivity & Specificity & $\begin{array}{c}\text { Diagnostic } \\
\text { accuracy }\end{array}$ & $\begin{array}{c}\text { Kappa } \\
\text { statistics }\end{array}$ & $p$ value \\
\hline Nuclear enlargement (cut off 3)50\% & $100.00 \%$ & $100.00 \%$ & $100.00 \%$ & 1.0000 & $<0.001$ \\
\hline Oval nucleus (cut off 2)25\% & $100.00 \%$ & $100.00 \%$ & $100.00 \%$ & 1.0000 & $<0.001$ \\
\hline Nuclear clearing (cut off 4)50\% & $62.80 \%$ & $97.70 \%$ & $80.23 \%$ & 0.6050 & $<0.001$ \\
\hline Papillae (cut off 2)1\% & $46.50 \%$ & $97.70 \%$ & $72.09 \%$ & 0.4420 & $<0.001$ \\
\hline Colloid (cut off 2) 50\% & $100.00 \%$ & $32.60 \%$ & $66.28 \%$ & 0.3260 & $<0.001$ \\
\hline Nuclear grooving (cut off 2)5\% & $41.90 \%$ & $95.30 \%$ & $68.60 \%$ & 0.3720 & $<0.001$ \\
\hline Nuclear crowding (cut off $>50 \%$ ) & $44.20 \%$ & $93.00 \%$ & $68.60 \%$ & 0.3720 & $<0.001$ \\
\hline
\end{tabular}




\section{Original Research Article}

From the above table it's clear that nuclear enlargement when diffuse ( $>50 \%)$, oval nucleus $(>25 \%)$, nuclear clearing $(>50 \%)$ are the best parameters followed by papillae, nuclear crowding, nuclear grooving, colloid status $(\mathrm{p}<0.001)$ are significant is differentiating benign from PTC cases with colloid status $(>50 \%)$ indicating towards benign nature of the lesion.

The scoring derived from our study was applied on 43 benign and 43 PTC cases. Maximum score was 15 and minimum zero. The below table describes the scoring of various benign thyroid lesions and PTC cases.

Table-5: Score applied on benign and PTC cases.

\begin{tabular}{|l|c|}
\hline Benign thyroid lesions & Score \\
\hline Adenomatous nodule & $0-6$ \\
\hline Follicular adenoma & $0-4$ \\
\hline Hurthle cell adenoma & $0-4$ \\
\hline Malignant lesions & Score \\
\hline PTC & $9-15$ \\
\hline FVPTC & $7-13$ \\
\hline
\end{tabular}

All benign cases scored less than six compared to malignant cases which scored more than seven. The classical variant of PTC showed higher score in contrast to FVPTC which scored low. However, it was $>6$ compared to benign cases.

\section{Discussion}

The main objective behind this study would be the overlapping features of benign and malignant lesions of thyroid posing difficulties in the diagnosis and ultimately affecting the treatment outcome. Unlike follicular carcinomas which require demonstration of vascular and capsular invasion, PTC diagnosis is based on nuclear features irrespective of invasion or metastasis although no single morphology will differentiate PTC from benign lesions [8].

The current study reviewed the presence, the percentage of tumor presenting with microscopic criteria of PTC and devised a score based on statistical analysis for the microscopic analysis of benign and PTC cases. This score included seven criteria with specific weight: colloid $<50 \%$ ( 0 when absent or 2 when present $)+$ papillae $>1 \%$ ( 0 when absent and 2 when present + nuclear crowding $>25 \%$ ( 0 when absent or 2 when present) + nuclear grooving $>5 \%$ ( 0 when absent or 2 when present) + nuclear enalrgement $>50 \%$ ( 0 when absent or 3 when present $)+$ oval nucleus $>25 \%$ ( 0 when absent and 2 when present $)+$ nuclear clearing $>50 \%(0$ when absent and 4 when present). The "gold standard" for the diagnosis of PTC still relies on microscopic features [9].

PTC, classical variant, as we know shows nuclear clearing, overcrowding, overlapping, abortive papillae. Clearing is said when nucleus shows peripheral condensation of chromatin giving it vesicular appearance and nuclear grooving when nucleus folds into itself [9]. About $70 \%$ of PTC cases in our study showed clearing followed by $41 \%$ of cases showing grooving. Nuclear grooving which is well appreciated on cytology specimens are also not specific to PTC cases and can be seen in adenomatous hyperplasia, Hashimoto's disease, diffuse hyperplasia etc [10]. This was reflected in our study were grooving was found to be a specific finding provided they are distributed diffusely. Ground glass appearance was not specific finding as it is seen in other benign lesions of thyroid such as nodular hyperplasia, Hashimotos thyroiditis etc $[10,11]$.

Nuclear pseudoinclusions which is specific to PTC cases is excluded in this study due to its close association with malignancy. Enlargement of the nucleus, ovoid nucleus are other features specific to PTC [10]. This enlargement results in over crowding. In our study, PTC cases showed nuclear enlargement ( $>50 \%$ area) with $93 \%$ showing ovoid nucleus. The Literature mentions about $90 \%$ of the PTC cases showing papillae, sticky colloid in $20 \%$ cases, psammoma bodies in $20-40 \%$ cases and nuclear grooving in $88 \%$ of the cases $[11,12]$. Many thyroid lesions can present with a papillary like features mimicking PTC and pose a diagnostic problem.

Benign lesions of thyroid like hylainizing trabecular neoplasms, benign papillary hyperplasia, lymphocytic thyroiditis and also FNA can have papillary like features. Hence, we have to be careful in identifying the complex branching papillary architecture of PTC from benign lesions. Apart from classical variant, follicular 


\section{Original Research Article}

variant of PTC never shows papillary architecture. Therefore, the diagnosis was purely based all the features in unison [3]. In our study, $44.2 \%$ cases showed well-formed papillary structures. There are several variants of PTC i.e follicular variant, tall cell variant, warthin like variant, oncocytic, columnar cell variant, diffuse sclerosing, clear cell etc [13]. Papillae are uncommonly seen in follicular variant and also the nuclear features are not pronounced in these cases. Histopathology plays an important role in diagnosing these cases. Very few studies have been done pertaining to methodical quantification of the individual feature of PTC cases in the past [14]. Hence in the present study, special emphasis has been placed to quantify and grade various features of PTC.

This study emphasises on morphometry as an objective tool to supplement subjective histopathology evaluation in differentiating benign from PTC cases. It's a quantitative analysis of various parameters i.e colloid, papillae, nuclear enlargement, nuclear grooving, oval nucleus and nuclear clearing. Over all assessment of these parameters helps to differentiate between benign and PTC, thus help in the definitive diagnosis of borderline cases. In our study, all the parameters that were considered were highly significant in differentiating benign from PTC cases ( $p$ value $<0.0001)$. Based on the scoring system proposed score $>7$ indicates malignancy with FVPTC on the lower side and classical variant of PTC on the upper side and benign cases less than 7 .

Usually, PTC cases are easily diagnosed based on nuclear features. However in cases of follicular variant of PTC were the classic features are subtle and also in cases of benign cases such as papillary hyperplasia of thyroid and adenomatous were nuclear features are pronounced, diagnosis becomes subjective and might result in diagnostic errors. Encapsulated variant of PTC has now renamed as non-invasive follicular thyroid neoplasm with papillary-like nuclear features" (NIFTP) due to its highly indolent behaviour $[8,15]$. In equivocal cases, terminology "well differentiated thyroid tumour of uncertain malignant potential" was proposed by Chernobyl pathologists for uncertain nuclear features [16].

In our study, we first analysed the percentage of various parameters proposed in benign and PTC cases followed by sensitivity and specificity with $p$ values to each cut off that was designed. In our study, the parameters were highly variable from cases to case and all the variables upon integration helped us to give an appropriate diagnosis. No PTC cases showed colloid status $>50 \%$ indicating that all the cases was within $50 \%$ status.
Nuclear enlargement, oval nucleus, nuclear clearing when combined together constituted $100 \%$ sensitivity and specificity when the distribution was diffuse $(>50 \%)$. Our results are comparable with those of Adeniran et al who also found that nuclear enlargement was present in a high proportion of tumours cells, optically clear nuclei in a slightly smaller proportion and nuclear grooves to a variable degree from case to case [17]. For these authors, the presence of nuclear pseudo inclusions was the less frequent finding among all tumours (54\%). Similar results were also seen in a study conducted by Verhulst et al who stressed the importance of ovoid nucleus, enlarged nuclei, dark staining colloid, lack of polarisation, nuclear grooves in determining malignant status of the lesions [9].

After determining the threshold values, it was observed that all the features were present in malignant cases, with nuclear features showing the upper hand. Nuclear grooving, ovoid nucleus and clearing when present diffusely showed almost $100 \%$ sensitivity and specificity. The importance of nuclear features in the diagnosis FVPTC was demonstrated by Devi et al [18] and Kunjumon et al [2], all of which were comparable to our study.

\section{Conclusion}

No previous attempts has been made in the past to score benign and malignant lesions of thyroid. The score proposed did help us to make a definite distinction between benign and PTC cases in routine practice when diagnosis was made upon integration.

Such a scaled score may be a useful tool in this field of thyroid pathology. The limitation of the study is that this score could be further tested by different teams of pathologists on different variants of PTC. This study shows that no single morphological feature is diagnostic of PTC and that a diagnosis is made upon the integration of various morphological features.

\section{Author contribution}

Concept and design: Dr. Swathi C Prabhu, Manuscript preparation: Dr. Swathi C. Prabhu, Dr. Umashankar T, Manuscript editing and manuscript review: Dr. Umashankar T.

Fndings: Nil; Conflict of Interest: None initiated Permission from IRB: Yes

\section{References}

1.DeLellis R, Lloyd R, Heitz P, Eng C. Pathology and genetics of tumours of endocrine organs. 3rd ed. Lyon: IARC Press; 2008. 


\section{Original Research Article}

2. Zidan J, Karen D, Stein M, et al. Pure versus follicular variant of papillary thyroid carcinoma: clinical features, prognostic factors, treatment, and survival. Cancer. 2003 Mar 1;97 (5):1181-5. DOI:10. 1002 /cncr. 11175

3. Kunjumon D, Upadhyaya K. Histopathological features of Papillary Thyroid Carcinoma with special emphasis on the significance of nuclear features in their diagnosis. Archives of Medicine and Health Sciences 2014; 2(1):16.

4. Unnikrishnan AG, Menon UV. Thyroid disorders in India: An epidemiological perspective. Indian $\mathrm{J}$ Endocrinol Metab. 2011 Jul;15 (Suppl 2):S78-81. doi: 10. 4103/2230-8210.83329.

5. Al-Brahim N, Asa S. Paillary thyroid carcinoma: an overview. Archives of pathology and laboratory medicine. 2019;130(7):1057-62. doi:10.1043/15432165 (2006)130[1057:PTCAO]2.0.CO;2

6. Rosai J. Papillary Thyroid Carcinoma: A Root-andBranch Rethink. American Journal of Clinical Pathology. 2008; 130(5):683-686. Available from: https://doi.org/10.1309/AJCPBF63BWMCYSLW

7. Fletcher C. Diagnostic histopathology of tumors. 3rd ed. [Edinburgh, Scotland]: Churchill Livingstone Elsevier; 2007. Chapter 18. Tumours of thyroid and parathyroid glands: 959-69.

8. Nikiforov YE, Seethala RR, Tallini G, et al. Nomenclature Revision for Encapsulated Follicular Variant of Papillary Thyroid Carcinoma: A Paradigm Shift to Reduce Overtreatment of Indolent Tumors. JAMA Oncol. 2016 Aug 1; 2 (8):1023-9. doi: 10.1001/ jamaoncol. 2016.0386.

9. Verhulst P, Devos P, Aubert S, Buob D, Cranshaw I, Do $\mathrm{Cao} C$ et al. A score based on microscopic criteria proposed for analysis of papillary carcinoma of the thyroid. Virchows Archiv 2008;452(3):233-240.
10. Rosai J, Ackerman L. Ackerman's surgical pathology. 9th ed. St. Louis: Mosby; 2004. Chapter 9. Thyroid gland;p.504-13.

11. LiVolsi VA. Papillary thyroid carcinoma: an update. Mod Pathol. 2011 Apr;24 Suppl 2:S1-9. doi: 10. 1038/ modpathol. 2010.129.

12. Jayaram G. Atlas and text of thyroid cytology. New Delhi: Arya Publications; 2006. Chapter 3. Papillary carcinoma: 35-49.

13. Lloyd RV, Buehler D, Khanafshar E. Papillary thyroid carcinoma variants. Head Neck Pathol. 2011 Mar; 5 (1):51-6. doi: 10.1007/s12105-010-0236-9. Epub 2011 Jan 8.

14. Sak SD. Variants of Papillary Thyroid Carcinoma: Multiple Faces of a Familiar Tumor. Turk Patoloji Derg. 2015;31 Suppl 1:34-47.

doi: 10.5146/tjpath.2015.01313.

15. Chan J. Strict criteria should be applied in the diagnosis of encapsulated follicular variant of papillary thyroid carcinoma. Am J Clin Pathol. 2002 Jan;117 (1): 16-8. DOI:10.1309/P7QL-16KQ-QLF4-XW0M

16. Williams ED. Guest Editorial: Two Proposals Regarding the Terminology of Thyroid Tumors. Int J Surg Pathol. 2000 Jul;8(3):181-183. DOI:10.1177/1066 89690000800304

17. Adeniran A, Chhieng D. Common Diagnostic Pitfalls in Thyroid Cytopathology. Cham: Springer International Publishing; 2016.

18. Padmavathi Devi C, Maruthi Devi K, Venugopal M, Partha Akarsh M. Nuclear Features in the Diagnosis of Follicular Variant of Papillary Thyroid CarcinomaThe Diagnostic Dilemma. International Journal of Otolaryngology and Head \&amp; Neck Surgery 2015; 04 (04): 296-302.

\section{How to cite this article?}

Prabhu S.C, Umashankar T. Scoring system for papillary thyroid carcinoma- a histopathological study. Trop J Path Micro 2018;4(8):610-616.doi: 10.17511/jopm.2018.i08.11. 